\title{
SOME RESULTS ON FUNCTION SPACES OF VARYING SMOOTHNESS
}

\author{
JAN SCHNEIDER \\ Max-Planck-Institute for Mathematics in the Science \\ Inselstrasse 22, 04103 Leipzig, Germany \\ E-mail: Jan.Schneider@mis.mpg.de
}

\begin{abstract}
This paper deals with function spaces of varying smoothness $B_{p}^{\mathbb{S}, s_{0}}\left(\mathbb{R}^{n}\right)$, where the function $\mathbb{S}: x \mapsto s(x)$ determines the smoothness pointwise. Those spaces were defined in [2] and treated also in [3]. Here we prove results about interpolation, trace properties and present a characterization of these spaces based on differences.
\end{abstract}

1. Introduction. The idea of function spaces where the smoothness can vary from point to point has quite a rich history. We described it in [2] and [3] with many references. There we presented our approach to a Besov-type function space of varying smoothness $B_{p}^{\mathbb{S}, s_{0}}\left(\mathbb{R}^{n}\right)$. We recall the definition here. Let us denote by $B_{x, r}$ the open ball in $\mathbb{R}^{n}$ centered at $x$ with radius $r>0$.

Definition 1. Let $1<p \leq \infty$ and let $\mathbb{S}: x \mapsto s(x)$ be a bounded lower semi-continuous function in $\mathbb{R}^{n}$ with $s_{\min } \geq s_{0}$ for a real number $s_{0}$. Then

$$
B_{p}^{\mathbb{S}, s_{0}}\left(\mathbb{R}^{n}\right)=\left\{f \in S^{\prime}\left(\mathbb{R}^{n}\right):\left\|f \mid B_{p}^{\mathbb{S}, s_{0}}\left(\mathbb{R}^{n}\right)\right\|<\infty\right\},
$$

normed by

$$
\left\|f\left|B_{p}^{s_{0}}\left(\mathbb{R}^{n}\right)\left\|+\sup _{K \in \mathbb{N}, x \in \mathbb{R}^{n}} 2^{-K\left(s_{K, x}-s_{0}\right)}\right\| f\right| B_{p}^{s_{K, x}}\left(B_{x, 2^{-K}}\right)\right\|
$$

where

$$
s_{K, x}=\inf _{|y-x| \leq 2^{-K+2}} s(y) .
$$

Here $s_{0} \leq s_{\min }=\inf _{y \in \mathbb{R}^{n}} s(y)$ plays the role of a global smoothness index but now complemented by the function $s(x)$, that gives for every point $x \in \mathbb{R}^{n}$ a possibly different

2000 Mathematics Subject Classification: Primary 46E35; Secondary 42B35.

Key words and phrases: Besov spaces, varying smoothness, interpolation, traces, characterization by differences.

Research of the author supported by Junior Research Team "Fractal Analysis", University of Jena, Germany.

The paper is in final form and no version of it will be published elsewhere. 
smoothness value. By $B_{p}^{s}\left(\mathbb{R}^{n}\right)$ we denote the usual Besov space $B_{p, q}^{s}\left(\mathbb{R}^{n}\right)$ in the case $p=q$ and by $B_{p}^{s}(\Omega)$ we mean its restriction to the domain $\Omega$.

Let us describe what happens in the norm (1). The first term checks the global smoothness $s_{0}$ of a given function $f$, whereas the supremum term concerns local improvements by the following procedure. For a fixed point $x \in \mathbb{R}^{n}$ we consider a ball centered in $x$ with radius $2^{-K}$ and ask if $f$ belongs to the Besov space with smoothness $s_{K, x} \geq s_{0}$ in this ball. Now we increase $K$ and therefore shrink the ball around $x$ and ask the same question again with respect to a possibly higher degree of smoothness. We continue this procedure for all $K$, then all $x$, and finally check if the supremum over all these norms multiplied by the weight factor $2^{-K\left(s_{K, x}-s_{0}\right)}$ is finite. This factor appears in a natural way when we compare Besov space norms on balls with different smoothness levels, for more details we refer to [2] and [3].

In [2] we proved that $B_{p}^{\mathbb{S}, s_{0}}\left(\mathbb{R}^{n}\right)$ is a Banach space and some basic properties for $s_{0}<1 / p$ like a pointwise multiplier assertion and an embedding theorem. One main result was a characterization by the following wavelet-expansion. An arbitrary function $f$ belonging to a Besov space can be written as

$$
f(x)=\sum_{l=0}^{2^{n}-1} \sum_{j=0}^{\infty} \sum_{m \in \mathbb{Z}^{n}} \lambda_{j, m}^{l}(f) \Psi^{l}\left(2^{j} x-m\right),
$$

where $\Psi^{l}$ are fixed functions with compact support and $\lambda_{j, m}^{l}(f)$ are complex numbers containing all the information about $f$, for details we refer to [8]. By means of this expansion we stated in [2] (section 5) the wavelet characterization of $B_{p}^{\mathbb{S}, s_{0}}\left(\mathbb{R}^{n}\right)$ with the help of weighted sequence spaces.

This result was the starting point for further investigations, for example, we proved an interesting connection to the so-called 2-microlocal spaces $C^{s, s^{\prime}}\left(x^{0}\right)$, see [2] (section $6.3)$ and [1] for more details.

In the case of negative smoothness the whole theory is published in [3]. For positive smoothness the theory is not yet satisfatory but we postpone this discussion to another paper.

In the next section we collect some new results on interpolation and traces of the spaces $B_{p}^{\mathbb{S}, s_{0}}\left(\mathbb{R}^{n}\right)$ which are not contained in [2] or [3]. In section 3 we discuss a characterization of $B_{p}^{\mathbb{S}, s_{0}}\left(\mathbb{R}^{n}\right)$ by differences. Now we list two older results on Besov spaces that we will need later on.

Proposition 2. Let $1<p \leq \infty,-\infty<s<1 / p$ and $0<\lambda \leq 1$, then

$$
\left\|f(\lambda \cdot)\left|B_{p}^{s}\left(B_{1}\right)\left\|\sim \lambda^{s-n / p}\right\| f\right| B_{p}^{s}\left(B_{\lambda}\right)\right\|,
$$

where the equivalence constants are independent of $f \in B_{p}^{s}\left(B_{\lambda}\right)$ and $\lambda$.

This has been formulated briefly in [7], section 5.20, p. 69, and proved in [6], 3.9(iii).

Proposition 3. Let $1<p \leq \infty, s \in \mathbb{R}$ and $0<\lambda \leq 1$, then

$$
\left\|f(\lambda \cdot)\left|B_{p}^{s}\left(B_{1}\right)\left\|\geq c \lambda^{s-n / p}\right\| f\right| B_{p}^{s}\left(B_{\lambda}\right)\right\|,
$$

where the equivalence constants are independent of $f$ and $\lambda$.

This has been stated as Proposition 2.4 in [2]. 
These two homogeneity properties were essential for the methods of the proofs in [2] and [3], they also will be important for our purpose in this paper.

2. Supplements to the space $B_{p}^{\mathbb{S}, s_{0}}\left(\mathbb{R}^{n}\right)$. In this section we treat two standard questions in the theory of function spaces, which are quite different from each other: interpolation and traces.

2.1. Interpolation. We restrict ourselves to the case $p_{1}=p_{2}=p$ and the same basic level of smoothness $s_{0}$ in both spaces of the interpolation couple $\left(B_{p}^{\mathbb{S}^{1}, s_{0}}\left(\mathbb{R}^{n}\right), B_{p}^{\mathbb{S}^{2}, s_{0}}\left(\mathbb{R}^{n}\right)\right)$. Then by means of the well-known K-method we can state the following.

THEOREM 4. Let $1<p<\infty$ and let $\mathbb{S}^{1}, \mathbb{S}^{2}$ be negative lower semi-continuous functions in $\mathbb{R}^{n}$ with $s_{0} \leq\left(s_{\min }^{1}, s_{\min }^{2}\right)$. Then

$$
\left(B_{p}^{\mathbb{S}^{1}, s_{0}}\left(\mathbb{R}^{n}\right), B_{p}^{\mathbb{S}^{2}, s_{0}}\left(\mathbb{R}^{n}\right)\right)_{\Theta, p} \hookrightarrow B_{p}^{\mathbb{S}, s_{0}}\left(\mathbb{R}^{n}\right),
$$

if $s(x)=(1-\Theta) s^{1}(x)+\Theta s^{2}(x)$ for $0<\Theta<1$.

Proof. Let $f \in\left(B_{p}^{\mathbb{S}^{1}, s_{0}}\left(\mathbb{R}^{n}\right), B_{p}^{\mathbb{S}^{2}}, s_{0}\left(\mathbb{R}^{n}\right)\right)_{\Theta, p}$ and

$$
\left\|f \mid\left(B_{p}^{\mathbb{S}^{1}, s_{0}}\left(\mathbb{R}^{n}\right), B_{p}^{\mathbb{S}^{2}, s_{0}}\left(\mathbb{R}^{n}\right)\right)_{\Theta, p}\right\|=\left(\int_{0}^{\infty} t^{-\Theta p} K(t, f)_{\mathbb{S}^{1}, \mathbb{S}^{2}}^{p} \frac{d t}{t}\right)^{1 / p},
$$

where the $K$-functional is defined by

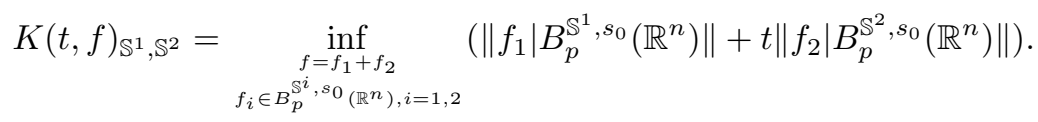

Now we estimate the $K$-functional

$$
\begin{aligned}
& K(t, f)_{\mathbb{S}^{1}, \mathbb{S}^{2}} \geq \inf _{\substack{f=f_{1}+f_{2} \\
f_{1}, f_{2} \in B_{p}^{s_{0}}\left(\mathbb{R}^{n}\right)}}\left(\left\|f_{1}\left|B_{p}^{s_{0}}\left(\mathbb{R}^{n}\right)\|+t\| f_{2}\right| B_{p}^{s_{0}}\left(\mathbb{R}^{n}\right)\right\|\right) \\
& +\inf _{\substack{f=f_{1}+f_{2} \\
\mathbb{S}^{i}, s_{0}}}\left(\sup _{x, K} 2^{-K\left(s_{K, x}^{1}-s_{0}\right)}\left\|f_{1} \mid B_{p}^{s_{K, x}^{1}}\left(B_{x, 2-K}\right)\right\|\right. \\
& \left.+t \sup _{x, K} 2^{-K\left(s_{K, x}^{2}-s_{0}\right)}\left\|f_{2} \mid B_{p}^{s_{K, x}^{2}}\left(B_{x, 2^{-K}}\right)\right\|\right) .
\end{aligned}
$$

The first infimum is the definition of $K(t, f)_{s_{0}}$ in obvious notation. The second infimum is estimated further with the help of homogeneity:

$$
\begin{aligned}
& \inf _{\substack{f=f_{1}+f_{2} \\
\mathbb{S}^{i}, s_{0}}} \sup _{\substack{\left.\mathbb{R}^{n}\right), K \\
f_{i} \in B_{p}}} 2^{-K\left(s_{K, x}^{1}-s_{0}\right)}\left\|f_{1} \mid B_{p}^{s_{K, x}^{1}}\left(B_{x, 2^{-K}}\right)\right\| \\
& \left.+t \sup _{x, K} 2^{-K\left(s_{K, x}^{2}-s_{0}\right)}\left\|f_{2} \mid B_{p}^{s_{K, x}^{2}}\left(B_{x, 2^{-K}}\right)\right\|\right) \\
& \geq c \sup _{x, K} 2^{-K\left(n / p-s_{0}\right)} \inf _{f=f_{1}+f_{2}}\left(\left\|f_{1}\left(2^{-K} \cdot\right)\left|B_{p}^{s_{K, x}^{1}}\left(B_{x, 1}\right)\|+t\| f_{2}\left(2^{-K} \cdot\right)\right| B_{p}^{s_{K, x}^{2}}\left(B_{x, 1}\right)\right\|\right) \\
& f_{i} \in B_{p}^{s_{K, x}^{i}}\left(B_{x, 2}-K\right), i=1,2 \\
& =c \sup _{x, K} 2^{-K\left(n / p-s_{0}\right)} K\left(t, f\left(2^{-K} \cdot\right)\right)_{s_{K, x}^{1}, s_{K, x}^{2}}
\end{aligned}
$$


again in obvious notation. Therefore, we get for the $K$-functionals

$$
K(t, f)_{\mathbb{S}^{1}, \mathbb{S}^{2}} \geq K(t, f)_{s_{0}}+c \sup _{x, K} 2^{-K\left(n / p-s_{0}\right)} K\left(t, f\left(2^{-K} \cdot\right)\right)_{s_{K, x}^{1}, s_{K, x}^{2}} .
$$

Inserting this estimate into the norm of the interpolation space, we have

$$
\begin{aligned}
& \left\|f \mid\left(B_{p}^{\mathbb{S}^{1}, s_{0}}\left(\mathbb{R}^{n}\right), B_{p}^{\mathbb{S}^{2}, s_{0}}\left(\mathbb{R}^{n}\right)\right)_{\Theta, p}\right\|^{p} \\
& \geq c \int_{0}^{\infty} t^{-\Theta p} K(t, f)_{s_{0}}^{p} \frac{d t}{t}+c \sup _{x, K} 2^{-K\left(n / p-s_{0}\right) p} \int_{0}^{\infty} t^{-\Theta p} K\left(t, f\left(2^{-K} \cdot\right)\right)_{s_{K, x}^{1}, s_{K, x}^{2}}^{p} \frac{d t}{t} \\
& =c\left\|f \mid\left(B_{p}^{s_{0}}\left(\mathbb{R}^{n}\right), B_{p}^{s_{0}}\left(\mathbb{R}^{n}\right)\right)_{\Theta, p}\right\|^{p} \\
& \quad+c \sup _{x, K} 2^{-K\left(n / p-s_{0}\right) p}\left\|f\left(2^{-K} \cdot\right) \mid\left(B_{p}^{s_{K, x}^{1}}\left(B_{x, 1}\right), B_{p}^{s_{K, x}^{2}}\left(B_{x, 1}\right)\right)_{\Theta, p}\right\|^{p} .
\end{aligned}
$$

By well known interpolation results on Besov spaces, see chapters 2.4.1. and 4.3.1. in [4], we can further write

$$
\begin{aligned}
\left\|f \mid\left(B_{p}^{\mathbb{S}^{1}, s_{0}}\left(\mathbb{R}^{n}\right), B_{p}^{\mathbb{S}^{2}, s_{0}}\left(\mathbb{R}^{n}\right)\right)_{\Theta, p}\right\|^{p} & \\
& \geq c\left\|f\left|B_{p}^{s_{0}}\left(\mathbb{R}^{n}\right)\left\|^{p}+c \sup _{x, K} 2^{-K\left(n / p-s_{0}\right) p}\right\| f\left(2^{-K} \cdot\right)\right| B_{p}^{s_{K, x}}\left(B_{x, 1}\right)\right\|^{p} .
\end{aligned}
$$

Finally, by homogeneity we arrive at

$$
\begin{aligned}
\left\|f \mid\left(B_{p}^{\mathbb{S}^{1}, s_{0}}\left(\mathbb{R}^{n}\right), B_{p}^{\mathbb{S}^{2}, s_{0}}\left(\mathbb{R}^{n}\right)\right)_{\Theta, p}\right\|^{p} & \\
& \geq c\left\|f\left|B_{p}^{s_{0}}\left(\mathbb{R}^{n}\right)\left\|^{p}+c \sup _{x, K} 2^{-K\left(s_{K, x}-s_{0}\right) p}\right\| f\right| B_{p}^{s_{K, x}}\left(B_{x, 2^{-K}}\right)\right\|^{p} \geq c\left\|f \mid B_{p}^{\mathbb{S}, s_{0}}\left(\mathbb{R}^{n}\right)\right\|^{p},
\end{aligned}
$$

which proves the theorem.

REMARK 5. The interpolation meets exactly our expectations and is a generalization of well-known classical results. Of course, it is desirable to have also the converse embedding, that would mean $\left(B_{p}^{\mathbb{S}^{1}, s_{0}}\left(\mathbb{R}^{n}\right), B_{p}^{\mathbb{S}^{2}, s_{0}}\left(\mathbb{R}^{n}\right)\right)_{\Theta, p}=B_{p}^{\mathbb{S}, s_{0}}\left(\mathbb{R}^{n}\right)$, but unfortunately the method of the above proof does not carry over to the opposite direction. The reason is that the infimum appearing in the definition of the $K$-functional can be moved inside of the norm in $B_{p}^{\mathbb{S}, s_{0}}\left(\mathbb{R}^{n}\right)$ only by making the term smaller.

2.2. Traces. Let us first provide an older result that is needed in this subsection.

Proposition 6. Let $1<p \leq \infty$ and $\mathbb{S}: x \mapsto s(x)$ be a bounded lower semi-continuous function in $\mathbb{R}^{n}$. Then for $K_{0} \in \mathbb{N}$

$$
\left\|f\left|B_{p}^{s_{0}}\left(\mathbb{R}^{n}\right)\left\|+\sup _{x \in \mathbb{R}^{n}} \sup _{K \geq K_{0}} 2^{-K\left(s_{K, x}-s_{0}\right)}\right\| f\right| B_{p}^{s_{K, x}}\left(B_{x, 2^{-K}}\right)\right\|
$$

is an equivalent norm in $B_{p}^{\mathbb{S}, s_{0}}\left(\mathbb{R}^{n}\right)$.

A proof of this proposition can be found in [3] (Proposition 3.7). It tells us that only large values of $K$, corresponding to small balls, are of interest in the norm of $B_{p}^{\mathbb{S}, s_{0}}\left(\mathbb{R}^{n}\right)$.

Now we are interested in the trace of a function $f$ on the hyperplane $y_{n}=0$, which we identify with $\mathbb{R}^{n-1}$. We define the operator $\operatorname{Tr}: f(y) \mapsto f\left(y^{\prime}, 0\right)$ with $y=\left(y^{\prime}, y_{n}\right) \in \mathbb{R}^{n}$, where the ' always indicates a coordinate in $\mathbb{R}^{n-1}$. For the expressions $s_{K, x}$ and $B_{x, r}$ we denote the $(n-1)$-dimensional counterparts with $\bar{s}_{K, x^{\prime}}$ and $\bar{B}_{x^{\prime}, r}$. 
THEOREM 7. Let $1<p \leq \infty$ and let $\mathbb{S}$ be a positive lower semi-continuous function in $\mathbb{R}^{n}$ with $s_{0}>n / p$. Then

$$
\operatorname{Tr}: B_{p}^{\mathbb{S}, s_{0}}\left(\mathbb{R}^{n}\right) \rightarrow B_{p}^{s\left(x^{\prime}, 0\right)-1 / p, s_{0}-1 / p}\left(\mathbb{R}^{n-1}\right)
$$

is a bounded linear operator if

$$
s\left(x^{\prime}, x_{n}\right) \geq s\left(x^{\prime}, 0\right) \quad \text { for all }\left|x_{n}\right| \leq \varepsilon \text { for an } \varepsilon>0 .
$$

REMARK 8. The condition we actually need in the following proof is

$$
s_{K, x^{\prime}}=\bar{s}_{K, x^{\prime}} \quad \text { for all } x^{\prime} \in \mathbb{R}^{n-1} \text { and all } K \geq K_{0} \in \mathbb{N} .
$$

But condition (4) is sufficient for that and means that the function $s(x)$ has a local minimum along the hyperplane $y_{n}=0$, which is not really natural but on the other hand not very restrictive.

Proof. We have to show

$$
\left\|f\left(y^{\prime}, 0\right)\left|B_{p}^{s\left(x^{\prime}, 0\right)-1 / p, s_{0}-1 / p}\left(\mathbb{R}^{n-1}\right)\|\leq c\| f\right| B_{p}^{\mathbb{S}, s_{0}}\left(\mathbb{R}^{n}\right)\right\| .
$$

The global part of (5) is clear by chapter 2.7.2 in [5]. The local part reads as follows

$$
\begin{aligned}
\sup _{K, x^{\prime}} 2^{-K\left(\bar{s}_{K, x^{\prime}}-s_{0}\right)}\left\|f\left(y^{\prime}, 0\right) \mid B_{p}^{\bar{s}_{K, x^{\prime}}-1 / p}\left(\bar{B}_{x^{\prime}, 2^{-K}}\right)\right\| & \\
& \leq c \sup _{x, K} 2^{-K\left(s_{K, x}-s_{0}\right)}\left\|f \mid B_{p}^{s_{K, x}}\left(B_{x, 2^{-K}}\right)\right\| .
\end{aligned}
$$

We start to estimate the norm on the left-hand side. By definition we have

$$
\left\|f\left(y^{\prime}, 0\right)\left|B_{p}^{\bar{s}_{K, x^{\prime}}-1 / p}\left(\bar{B}_{x^{\prime}, 2^{-K}}\right)\|=\inf \| g\right| B_{p}^{\bar{s}_{K, x^{\prime}}-1 / p}\left(\mathbb{R}^{n-1}\right)\right\|,
$$

where the infimum is taken over all functions $g$ with $\left.g\right|_{\bar{B}_{x^{\prime}, 2^{-K}}}=f\left(y^{\prime}, 0\right)$. By the well known trace result for Besov spaces (we refer again to chapter 2.7.2. in [5]) we know that for every such function $g$ there is an extended function $G$ living on $\mathbb{R}^{n}$ with

$$
\left\|g\left|B_{p}^{\bar{s}_{K, x^{\prime}}-1 / p}\left(\mathbb{R}^{n-1}\right)\|\leq c\| G\right| B_{p}^{\bar{s}_{K, x^{\prime}}}\left(\mathbb{R}^{n}\right)\right\| .
$$

Because of our assumption (4) together with Remark 9 we can write $s_{K, x^{\prime}}$ instead of $\bar{s}_{K, x^{\prime}}$ on the right-hand side for large $K$. If we now restrict the infimum in (7) only to those functions $g$ such that for the corresponding extended function $G$ the equality $\left.G\right|_{B_{x^{\prime}, 2-K}}=f$ holds, which we denote by $*$, then we find

$$
\left\|f\left(y^{\prime}, 0\right)\left|B_{p}^{\bar{s}_{K, x^{\prime}}-1 / p}\left(\bar{B}_{x^{\prime}, 2^{-K}}\right)\left\|\leq c \inf _{*}\right\| G\right| B_{p}^{s_{K, x^{\prime}}}\left(\mathbb{R}^{n}\right)\right\|=c\left\|f \mid B_{p}^{s_{K, x^{\prime}}}\left(B_{x^{\prime}, 2^{-K}}\right)\right\| .
$$

Inserting this into the left-hand side of (6) we finally get with Proposition 6

$$
\begin{aligned}
\sup _{K, x^{\prime}} 2^{-K\left(\bar{s}_{K, x^{\prime}}-s_{0}\right)} \| f\left(y^{\prime}, 0\right) \mid B_{p}^{\bar{s}_{K, x^{\prime}}-1 / p} & \left(\bar{B}_{\left.x^{\prime}, 2^{-K}\right)}\right) \\
& \leq c \sup _{K>K_{0}, x^{\prime}} 2^{-K\left(\bar{s}_{K, x^{\prime}}-s_{0}\right)}\left\|f \mid B_{p}^{s_{K, x^{\prime}}}\left(B_{x^{\prime}, 2^{-K}}\right)\right\| \\
& \leq c \sup _{K>K_{0}, x^{\prime}} 2^{-K\left(s_{K, x^{\prime}}-s_{0}\right)}\left\|f \mid B_{p}^{s_{K, x^{\prime}}}\left(B_{x^{\prime}, 2^{-K}}\right)\right\| \\
& \leq c \sup _{K, x} 2^{-K\left(s_{K, x}-s_{0}\right)}\left\|f \mid B_{p}^{s_{K, x}}\left(B_{x, 2}-K\right)\right\|,
\end{aligned}
$$

where we used $s_{K, x^{\prime}}=\bar{s}_{K, x^{\prime}}$ for large $K$ again. This is the desired inequality (6). 
REMARK 9. The condition $s_{0}>n / p$ in Theorem 8 stands somehow in contrast to the condition $s_{0}<1 / p$ which appears almost in the whole theory because in the proofs the homogeneity property (2) is always needed, see [2]. But talking about traces we need a continuous representative in $B_{p}^{\mathbb{S}, s_{0}}\left(\mathbb{R}^{n}\right)$ and so the range of parameters is restricted to $s>n / p$. That means that the Theorem is in some sense still isolated from the rest of the theory.

REMARK 10. To prove that $B_{p}^{s\left(x^{\prime}, 0\right)-1 / p, s_{0}-1 / p}\left(\mathbb{R}^{n-1}\right)$ is the exact trace space, we have to show that there exists a bounded linear extension operator

$$
\text { Ext }: B_{p}^{s\left(x^{\prime}, 0\right)-1 / p, s_{0}-1 / p}\left(\mathbb{R}^{n-1}\right) \rightarrow B_{p}^{\mathbb{S}, s_{0}}\left(\mathbb{R}^{n}\right) .
$$

Unfortunately, no such result is available yet.

3. Characterization by differences. In [2] we developed the theory of $B_{p}^{\mathbb{S}, s_{0}}\left(\mathbb{R}^{n}\right)$ for general smoothness. But it turned out that for positive smoothness the arguments were not straightforward. We always had to compensate the lack of homogeneity for the Besov space norm. H.-G. Leopold suggested substituting the local term in (1) by a semi-norm based on differences in such a way that the homogeneity would be provided for positive smoothness. To present the essence of this idea is the aim of this section.

3.1. Preliminaries. First we make some preparations and fix the notation here. For $h \in \mathbb{R}$ and $M \in \mathbb{N}$ we put

$$
\Delta_{h}^{M, i} f(x)=\sum_{l=0}^{M}\left(\begin{array}{c}
M \\
l
\end{array}\right)(-1)^{l} f\left(x+(M-l) h e_{i}\right)
$$

and set

$$
\Delta_{h}^{M, i} f(x, \Omega)= \begin{cases}\Delta_{h}^{M, i} f(x), & x+(M-l) h e_{i} \in \Omega \forall l=0, \ldots, M, \\ 0, & \text { otherwise, }\end{cases}
$$

for a domain $\Omega$ in $\mathbb{R}^{n}$. Now we define the following semi-norm for $s<M$ :

$$
\left\|f \mid B_{p}^{s}(\Omega)\right\|_{\Delta^{M}}=\left(\sum_{i=1}^{n} \int_{|h|}|h|^{-s p}\left\|\Delta_{h}^{M, i} f(\cdot, \Omega) \mid L_{p}(\Omega)\right\|^{p} \frac{d h}{|h|}\right)^{1 / p} .
$$

Here the integration is meant to be over $|h| \leq \operatorname{dist}(\Omega)$. We denote by $B_{x, r}$ the ball with radius $r>0$ centered at $x \in \mathbb{R}^{n}$. In the case $x=0$ we omit it and write only $B_{r}$.

Proposition 11. Let $1<p \leq \infty$ and $M>s>0$, then

$$
\left\|f(\lambda \cdot)\left|B_{p}^{s}\left(B_{1}\right)\left\|_{\Delta^{M}}=\lambda^{s-n / p}\right\| f\right| B_{p}^{s}\left(B_{\lambda}\right)\right\|_{\Delta^{M}} .
$$

Proof. We prove the assertion for $M=1$ because for differences of higher order the proof is analogous. Putting $f(\lambda \cdot)=g$ we have $\Delta_{k} g(y)=\Delta_{\lambda k} f(\lambda y)$. Now we caculate with $\lambda k=h$

$$
\begin{aligned}
\left\|f(\lambda \cdot) \mid B_{p}^{s}\left(B_{1}\right)\right\|_{\Delta}^{p} & =\sum_{i=1}^{n} \int_{|k| \leq 2}|k|^{-s p}\left\|\Delta_{k}^{i} g\left(\cdot, B_{1}\right) \mid L_{p}\left(B_{1}\right)\right\|^{p} \frac{d k}{|k|} \\
& =\sum_{i=1}^{n} \int_{|k| \leq 2}|k|^{-s p}\left\|\Delta_{\lambda k}^{i} f\left(\lambda \cdot, B_{1}\right) \mid L_{p}\left(B_{1}\right)\right\|^{p} \frac{d k}{|k|}
\end{aligned}
$$




$$
\begin{aligned}
& =\sum_{i=1}^{n} \int_{|k| \leq 2}|k|^{-s p} \lambda^{-n}\left\|\Delta_{\lambda k}^{i} f\left(\cdot, B_{\lambda}\right) \mid L_{p}\left(B_{\lambda}\right)\right\|^{p} \frac{d k}{|k|} \\
& =\sum_{i=1}^{n} \int_{\left|\lambda^{-1} h\right| \leq 2}\left|\lambda^{-1} h\right|^{-s p} \lambda^{-n}\left\|\Delta_{h}^{i} f\left(\cdot, B_{\lambda}\right) \mid L_{p}\left(B_{\lambda}\right)\right\|^{p} \lambda^{-1} \frac{d h}{\left|\lambda^{-1} h\right|} \\
& =\lambda^{s p-n} \sum_{i=1}^{n} \int_{|h| \leq 2 \lambda}|h|^{-s p}\left\|\Delta_{h}^{i} f\left(\cdot, B_{\lambda}\right) \mid L_{p}\left(B_{\lambda}\right)\right\|^{p} \frac{d h}{|h|} \\
& =\lambda^{s p-n}\left\|f \mid B_{p}^{s}\left(B_{\lambda}\right)\right\|_{\Delta}^{p} .
\end{aligned}
$$

Inspecting the proof one easily verifies that

$$
\left\|f(\lambda \cdot)\left|B_{p}^{s}\left(\mathbb{R}^{n}\right)\left\|_{\Delta^{M}}=\lambda^{s-n / p}\right\| f\right| B_{p}^{s}\left(\mathbb{R}^{n}\right)\right\|_{\Delta^{M}}
$$

holds as well.

We add here the following fact because we shall need it in the sequel.

Proposition 12. Let $1<p \leq \infty, s>0, s \geq s_{0} \in \mathbb{R}$ and $\Omega \subseteq \mathbb{R}^{n}$. Then

$$
\left\|f\left|B_{p}^{s}(\Omega)\|\sim\| f\right| B_{p}^{s_{0}}(\Omega)\right\|+\left\|f \mid B_{p}^{s}(\Omega)\right\|_{\Delta^{M}} .
$$

The proof is left to the reader.

3.2. Equivalence theorem. First we need an older equivalence assertion to prove the new one.

THEOREM 13. Let $1<p \leq \infty$ and $\mathbb{S}: x \mapsto s(x)$ be a bounded lower semi-continuous function in $\mathbb{R}^{n}$ with $s_{\max }-m<1 / p$ for a natural number $m$. Then for $s_{0}<1 / p$

$$
\left\|f\left|B_{p}^{s_{0}}\left(\mathbb{R}^{n}\right)\left\|+\sup _{K \in \mathbb{N}, x \in \mathbb{R}^{n}} 2^{-K\left(s_{K, x}-s_{0}\right)} \sum_{|\alpha|=m}\right\| D^{\alpha} f\right| B_{p}^{s_{K, x}-m}\left(B_{x, 2^{-K}}\right)\right\|
$$

is an equivalent norm in $B_{p}^{\mathbb{S}, s_{0}}\left(\mathbb{R}^{n}\right)$.

This was proved in [2], section 3.2.

Now we provide our main result in this section and discuss its history afterwards.

THEOREM 14. Let $1<p \leq \infty$ and let $\mathbb{S}$ be a positive lower semi-continuous function in $\mathbb{R}^{n}$ with $s_{0}<1 / p$. Then

$$
\left\|f\left|B_{p}^{s_{0}}\left(\mathbb{R}^{n}\right)\left\|+\sup _{K, x} 2^{-K\left(s_{K, x}-s_{0}\right)}\right\| f\right| B_{p}^{s_{K, x}}\left(B_{x, 2^{-K}}\right)\right\|_{\Delta^{M}}
$$

is an equivalent norm in $B_{p}^{\mathbb{S}, s_{0}}\left(\mathbb{R}^{n}\right)$.

Proof. We have to handle the supremum term only. For one direction we start with fomula (3) and get by (10)

$$
\begin{aligned}
\left\|f \mid B_{p}^{s_{K, x}}\left(B_{x, 2^{-K}}\right)\right\| \\
\quad \leq c 2^{K\left(s_{K, x}-n / p\right)}\left\|f\left(2^{-K} \cdot\right) \mid B_{p}^{s_{K, x}}\left(B_{x, 1}\right)\right\| \\
\leq c 2^{K\left(s_{K, x}-n / p\right)}\left(\left\|f\left(2^{-K} \cdot\right)\left|B_{p}^{s_{0}}\left(B_{x, 1}\right)\|+\| f\left(2^{-K} \cdot\right)\right| B_{p}^{s_{K, x}}\left(B_{x, 1}\right)\right\|_{\Delta^{M}}\right) .
\end{aligned}
$$

Now the formulas (2) and (8) provide homogeneity for both terms in the brackets and we arrive at the desired estimate for one direction. For the opposite direction we start with formula (8) and get by (10) 


$$
\begin{aligned}
\left\|f \mid B_{p}^{s_{K, x}}\left(B_{x, 2^{-K}}\right)\right\|_{\Delta^{M}} & \leq c 2^{K\left(s_{K, x}-n / p\right)}\left\|f\left(2^{-K} \cdot\right) \mid B_{p}^{s_{K, x}}\left(B_{x, 1}\right)\right\|_{\Delta^{M}} \\
& \leq c 2^{K\left(s_{K, x}-n / p\right)}\left\|f\left(2^{-K} \cdot\right) \mid B_{p}^{s_{K, x}}\left(B_{x, 1}\right)\right\| .
\end{aligned}
$$

Now we are in the same situation as in the proof of Theorem 13, see step 2 of the proof of Theorem 3.2 in [2]. Just like there we arrive by homogeneity at

$$
\begin{aligned}
& \left\|f \mid B_{p}^{s_{K, x}}\left(B_{x, 2^{-K}}\right)\right\|_{\Delta^{M}} \\
& \quad \leq c 2^{K\left(s_{K, x}-s_{0}\right)}\left\|f\left|B_{p}^{s_{0}}\left(\mathbb{R}^{n}\right)\left\|+c \sum_{|\alpha|=m}\right\| D^{\alpha} f\right| B_{p}^{s_{K, x}-m}\left(B_{x, 2^{-K}}\right)\right\|
\end{aligned}
$$

and with Theorem 13 can conclude

$$
\begin{aligned}
& \sup _{K, x} 2^{-K\left(s_{K, x}-s_{0}\right)}\left\|f \mid B_{p}^{s_{K, x}}\left(B_{x, 2^{-K}}\right)\right\|_{\Delta^{M}} \\
& \quad \leq c\left\|f\left|B_{p}^{s_{0}}\left(\mathbb{R}^{n}\right)\left\|+c \sup _{K, x} 2^{-K\left(s_{K, x}-s_{0}\right)} \sum_{|\alpha|=m}\right\| D^{\alpha} f\right| B_{p}^{s_{K, x}-m}\left(B_{x, 2^{-K}}\right)\right\| \\
& \leq c\left\|f\left|B_{p}^{s_{0}}\left(\mathbb{R}^{n}\right)\left\|+c \sup _{K, x} 2^{-K\left(s_{K, x}-s_{0}\right)}\right\| f\right| B_{p}^{s_{K, x}}\left(B_{x, 2^{-K}}\right)\right\|,
\end{aligned}
$$

which is the second direction.

One can take the finiteness of (12) for the definition of a space of varying smoothness, say $\stackrel{\Delta}{B}_{p}^{\mathbb{S}, s_{0}}\left(\mathbb{R}^{n}\right)$. Now one can ask whether the results in [2] for positive smoothness can also be obtained for this space directly, because the local part of the norm defined by differences is easier to handle than the Fourier-analytical one defined via restriction. One goal would be to prove a wavelet characterization as in Theorem 6.3 in [2] without any restrictions on $s_{0}$. In fact, the homogeneity (8) make things much simpler, but the proofs would often have to use the equivalence (10), consequently they need also the homogeneity of the global term in (12) and therefore, by (2), we still must keep the condition $s_{0}<1 / p$

in crucial cases. That is the reason why the space $\stackrel{\Delta}{B}_{p}^{\mathbb{S}, s_{0}}\left(\mathbb{R}^{n}\right)$ would not yet yield an essential progress for the theory of varying smoothness.

Acknowledgements. The author thanks Prof. H.-G. Leopold and Prof. D. D. Haroske (both University of Jena) for their support.

\section{References}

[1] S. Jaffard and Y. Meyer, Wavelet methods for pointwise regularity and local oscillations of functions, Mem. Amer. Math. Soc. 123 (1996), No. 587.

[2] J. Schneider, Function spaces with varying smoothness, PhD Thesis, Jena, 2005.

[3] J. Schneider, Function spaces of varying smoothness I, Mathematische Nachrichten 280 (2007), 1801-1826.

[4] H. Triebel, Interpolation Theory, Function Spaces, Differential Operators, Deutsch. Verl. Wiss., Berlin, and North-Holland, Amsterdam, 1978.

[5] H. Triebel, Theory of Function Spaces, Geest \& Portig, Leipzig, and Birkhäuser, Basel, 1983. 
[6] H. Triebel, Decompositions of function spaces, in: Progr. Nonl. Diff. Eq. Appl. 35 (1999), 691-730.

[7] H. Triebel, The Structure of Functions, Birkhäuser, Basel, 2001.

[8] H. Triebel, A note on wavelet bases in function spaces, in: Banach Center Publications 64, Warszawa, 2004, 193-206. 
